Z. Epileptol. 2020 33:30-36 https://doi.org/10.1007/s10309-019-00303-w Published online: 23 December 2019

(c) The Author(s) 2019

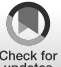

Victor Schmidbauer · Silvia Bonelli

Department of Neurology, Medical University of Vienna, Vienna, Austria

\section{Brief introduction}

Recent advances in neuroimaging and in particular in the field of functional imaging resulted in functional magnetic resonance imaging (fMRI) being the method of choice for noninvasively identifying the eloquent cortex during the individual presurgical work-up of patients with temporal lobe epilepsy (TLE). Primarily, the assessment of cognitive functions has high priority, in order to keep postsurgical restrictions as minimal as possible. Moreover, functional connectivity (FC) analysis and very recently connectome analyses represent highly promising imaging methods for identifying not only activation patterns during cognitive tasks but also underlying cognitive networks.

\section{Background}

Cognitive impairment is a frequent comorbidity in focal epilepsies and has a major impact on quality of life. Cognitive deficits either can result from the underlying disease or can be caused by adverse effects of antiepileptic drugs (AEDs). In TLE, representing the most common type of focal epilepsy, owing to anatomical structures located in the temporal lobe being affected, i.e., the hippocampus, parahippocampal gyrus and the amygdala, impairment of episodic memory functions is commonly observed. However, also impairment in language functions, particularly naming difficulties, have been reported [14].

In patients with medically refractory TLE, anterior temporal lobe resection (ATLR) is an effective and safe treatment option, leading to seizure remis- sion in up to $50-70 \%$ of these patients [9]. Comprehensive presurgical assessment is performed to identify the epileptic brain tissue that has to be removed for the patient to become seizure-free, and to predict the cognitive cost of this approach [30]. Cognitive deficits such as language and memory impairment have to be avoided. In recent years, epilepsy surgery has been carried out earlier in the course of the disease, and the potential benefits must be carefully weighed against the potential risks of decline. In order to successfully identify brain areas that are responsible for motor, language, and memory functions (eloquent cortex) and guide resection, a range of imaging techniques such as fMRI and diffusion tensor imaging (DTI) have been employed.

Functional MRI has proven a valid and reliable tool to investigate cognitive functions noninvasively during presurgical assessment [20], and recently it has increasingly replaced invasive procedures such as the Wada test. Compared with baseline neuropsychological assessment, it further has the potential of providing additional information regarding lateralization and localization of cognitive functions and particularly allows for the evaluation of functional reorganization processes over time. There is an increasing interest in its possible role for prediction of postoperative cognitive changes.

Functional MRI, like most other noninvasive imaging tools, is an activationbased method. The rationale behind this is that if a certain cognitive function is used, relevant brain areas will be activated. Blood flow will increase in these areas to compensate for the higher demand of oxygen. In fMRI, this is displayed by the BOLD (blood-oxygenlevel-dependent) contrast that represents the regional changes in blood flow over time. To obtain a reasonable temporal resolution, echo planar imaging (EPI), a fast MRI sequence, is used. Functional MRI has a high spatial resolution that, in principal, allows for very good localization of areas in the brain that are involved in certain tasks. However, which areas will be activated depends on the fMRI paradigm. Also, methodological limitations of fMRI techniques especially in the temporal lobes, such as lower MRI signal-to-noise ratio due to susceptibility artifacts and signal loss in areas that are close to larger blood vessels and bone tissue, have to be taken into account when interpreting the results.

In this review we discuss recent developments and future perspectives of fMRI such as FC analysis and connectomics in epilepsy and offer practical recommendations for clinical use of fMRI in assessing language and memory functions in TLE.

\section{Language}

\section{Assessing language functions using fMRI in epilepsy}

During evaluation for epilepsy surgery, fMRI is the most frequently applied noninvasive method for the visualization of language functions and offers a reliable alternative to the Wada test [13]. The primary aims of preoperative language fMRI are lateralization and localization of language functions and to use this information to predict postoperative complications and advise patients ac- 
Table 1 Recommended fMRI language paradigms (according to Black et al. 2017 [1])

\begin{tabular}{lll}
\hline Paradigm & Primarily activated area & Recommended in \\
\hline $\begin{array}{l}\text { Sentence completion } \\
\text { Word generation }\end{array}$ & Wernicke $>$ Broca & Adults $^{\mathrm{a}}$, Children $^{\mathrm{a}}$ \\
\hline $\begin{array}{l}\text { Rhyming } \\
\text { Object naming }\end{array}$ & Broca $>$ Wernicke & Adults $^{\mathrm{a}}$ \\
\hline $\begin{array}{l}\text { Antonym generation } \\
\text { Passive story listening }\end{array}$ & Broca $>$ Wernicke & Adults $^{\mathrm{b}}$, Children $^{\mathrm{a}}$ \\
\hline $\begin{array}{l}\text { Broca }>\text { Wernicke } \\
\text { Chighly recommended }\end{array}$ & Wernicke $>$ Broca & Children $^{\mathrm{b}}$ \\
\hline${ }^{\mathrm{b}}$ Complementary recommended & \\
\hline
\end{tabular}

cordingly. Clinically applied paradigms focus on classic language areas such as Broca's area (inferior frontal gyrus[IFG]) and Wernicke's area (superior temporal gyrus, supramarginal gyrus [SMG, STG]). Studies comparing language fMRI with the classic invasive methods (Wada test, electrocortical stimulation mapping [ESM]) showed that fMRI was a valid method for identifying the languagedominant hemisphere [16, 44]. Since language skills are composed of several components, i.e., semantics, pragmatics, and syntax, a single task is not enough for thorough assessment [5]. Furthermore, a language paradigm needs to fulfill specific criteria in order to provide appropriate results [5]: The task should be challenging without overtaxing the patient. Secondly, a balance of specificity and sensitivity for language-related activation should be provided by the task. Thirdly, the task should have the ability to determine intrahemispheric localization and interhemispheric lateralization of receptive as well as expressive language areas.

In clinical routine, block design paradigms are usually applied as they are robust and reliable compared with event-related designs [35]. The American Society of Functional Neuroradiology (ASFN) proposes default task batteries for adults and pediatric patients, and in order to get optimal data for the presurgical evaluation, at least two language paradigms should be used [5].

\section{Language $\mathrm{fMRI}$ in adults}

The Sentence Completion Task (SC), Word Generation Task (WG) complemented by the Rhyming Task are used. In case of cognitively impaired patients, the Object-Naming Task (ON) or the Pas- sive Story-Listening Task (PSL) should be applied. Instead of using a third paradigm, SC or WG can be repeated.

\section{Language $\mathrm{fMRI}$ in pediatric patients}

The SC, Rhyming Task, or PSL are used. The Antonym Generation Task (AG) should be selected as complementary task. However, in pediatric patients, age and the individual reading abilities need to be considered. The SC and WG task are considered the most frequently used language fMRI paradigms, whereas the other tasks are applied rather complementary.

- Table 1 gives an overview of currently recommended fMRI language paradigms.

About $90 \%$ of the healthy population show left-hemispheric dominance for language. About $10 \%$ are classified as having atypical language representation eral). In TLE patients, the incidence of atypical language representation is higher (78\% left-lateralized, $16 \%$ bilateral, 6\% right-lateralized). Frequently occurring seizures in childhood appear to severely affect the development of language-related areas and underlying networks. This interferes with reorganization processes and leads to a poor postsurgical outcome. Hence, early surgical interventions may contribute to an unimpaired development of language functions and should be considered in cases of intractable childhood epilepsy [21]. (right-hemispheric dominance, bilat-

\section{Localization of language function and prediction of postoperative deficits}

While language lateralization in TLE using fMRI is well established in many centers, reliable localization of language areas with $\mathrm{fMRI}$ is still not applicable in clinical routine testing [34]. This still requires invasive electrocortical stimulation mapping (ESM). First of all, test-retest series have shown that the localization of areas that were activated during a specific language fMRI task was less reliable than lateralization [11]. Furthermore, ESM studies showed only imperfect overlap with activation clusters of fMRI: In some cases electric stimulation of fMRI-activated brain areas did not result in language disturbances, while in others, crucial areas were not displayed during $\mathrm{fMRI}$ [17]. The differences may be related either to the applied language paradigms or to the statistical thresholds.

To date, language fMRI localization is not yet suitable for resection decision [12], but the use of combined fMRI paradigms targeting both expressive (WG, AG, ON) and receptive (SC, PSL) language-related areas may be helpful in planning electrode placement for ESM [10].

After ATLR of the language-dominant hemisphere, subtle word finding and naming difficulties are observed in approximately $50 \%$ of patients, whereas aphasia is rare [8]. Inleft TLE, a predictive value of fMRI language lateralization for naming decline $[7,31]$ could be shown. A recent fMRI study showed that auditory and visual naming fMRI tasks reliably activated posterior and basal temporal lobe regions that were strongly related to clinical naming performance [37]. Using these specific naming paradigms, postoperative naming deficits could be predicted with a positive predictive value of 91.6\% (100\% sensitivity and 92\%; [38]).

However, prediction models on an individual level are not yet sufficient to be applied in clinical routine. 


\section{Memory}

\section{Assessing memory functions using fMRI in epilepsy}

Memory impairment is common in patients with epilepsy. Working and longterm memory (autobiographical, verbal, visual memory) may become affected in a material-specific way, based on the site of lesion. Functional MRI can reveal memory networks noninvasively and reliably, and also the effect of surgery on these networks.

\section{Lateralization and localization of memory functions in TLE}

Patients with unilateral TLE often present with memory impairment that is specific to certain materials (e.g., verbal and visual). After temporal lobe surgery of the language-dominant hemisphere, more often verbal memory decline can be observed, while TL surgery in the nondominant hemisphere is more likely to result in visual-spatial memory decline. Many fMRI studies demonstrated material-specific lateralization of memory function in prefrontal but also medial-temporal regions ([23]; • Fig. 1). For clinical purposes, usually paradigms are applied that show bilateral MTL activation in healthy controls $[6,25]$. Previous fMRI studies in patients reported reduced activation in the TL ipsilateral to the seizure onset. The reduced activation within the affected TL but the increased contralateral MTL activation during memory fMRI has provided further evidence of the reorganization of memory function in TLE $[6,25,28,32]$. Still, it is a matter of debate whether reorganization toward the healthy hemisphere is effective, and whether it may be protective for memory decline after surgery. By correlation of fMRI activation and performance on standard neuropsychological memory tests it has been shown that higher MTL activation ipsilateral to the pathology was associated with better memory performance while contralateral, compensatory activation correlated with poorer performance $[6,25]$.

Methodologically, memory fMRI studies applying block designs have the

Z. Epileptol. 2020 33:30-36 https://doi.org/10.1007/s10309-019-00303-w

(c) The Author(s) 2019

\section{Schmidbauer $\cdot$ S. Bonelli}

\section{Recent developments in cognitive fMRI for temporal lobe epilepsy}

\section{Abstract}

Epilepsy is frequently accompanied by severe cognitive side effects. Temporal lobe epilepsy (TLE), and even successful surgical treatment, may affect cognitive function, in particular language as well as verbal and visual memory function. Epilepsy arising from the temporal lobe can be controlled surgically in up to $70 \%$ of patients. The goals of epilepsy surgery are to remove the brain areas generating the seizures without causing or aggravating neuropsychological deficits. This requires accurate localization of the brain areas generating the seizures ("epileptogenic zone") and the areas responsible for motor and cognitive functions, such as language and memory ("essential brain regions") during presurgical evaluation. In the past decades, functional magnetic resonance imaging (fMRI) has been increasingly used to noninvasively lateralize and localize not only primary motor and somatosensory areas, but also brain areas that are involved in everyday language and memory processes. The imaging modality also shows potential for predicting the effects of temporal lobe resection on language and memory function. Together with other MRI modalities, cognitive fMRI is a promising tool to improve surgical strategies tailored to individual patients with regard to functional outcome, by virtue of definition of epileptic cerebral areas that need to be resected and eloquent areas that need to be spared.

The aim of this review is to provide an overview of recent developments and practical recommendations for the clinical use of cognitive fMRI in TLE.

Keywords

Episodic memory - Language function . Reorganization - Anterior temporal lobe resection - Functional connectivity

\section{Aktuelle Entwicklungen in der kognitiven fMRT bei Temporallappenepilepsie}

\section{Zusammenfassung}

Epilepsie geht häufig mit schweren kognitiven Störungen einher. Die Temporallappenepilepsie (TLE) und auch eine erfolgreiche chirurgische Behandlung kann die kognitive Funktion beeinträchtigen, insbesondere Sprach- sowie verbale und visuelle Gedächtnisfunktionen. Bei bis zu $70 \%$ aller Patienten mit TLE kann chirurgisch Anfallsfreiheit erreicht werden. Ziel eines solchen Eingriffs ist es, die Areale des Gehirns, von denen die Anfälle ausgehen („epileptogene Zone“), zu entfernen, ohne neuropsychologische Beeinträchtigungen zu verursachen oder zu verstärken. Im Rahmen eines sorgfältigen präoperativen Monitorings ist es notwendig, sowohl die epileptogene Zone als auch die Areale, die für motorische und kognitive Funktionen verantwortlich sind (,essenzielle Hirnareale") möglichst genau zu lokalisieren. Die funktionelle Magnetresonanztomographie (fMRT) wurde in den letzten Dekaden zunehmend zur nichtinvasiven Lateralisation und Lokalisation von primären motorischen und somatosensorischen Bereichen, aber auch von Hirnarealen mit Beteiligung an Sprach- und Gedächtnisprozessen eingesetzt. Die fMRT hat auch das Potenzial, die Wirkungen der Temporallappenresektion auf Sprach- und Gedächtnisfunktionen zu prognostizieren. In Kombination mit anderen MRT-Verfahren ist die kognitive fMRT ein vielversprechendes Werkzeug für die Verbesserung chirurgischer Strategien, die in Bezug auf das funktionelle Ergebnis auf den individuellen Patienten zugeschnitten sind, indem zu resezierende epileptogene Hirnareale sowie zu schonende Sprachareale definiert werden. Ziel dieser Übersichtsarbeit ist es, jüngste Entwicklungen im Bereich der kognitiven fMRT bei TLE zusammenzufassen und praktische Empfehlungen für den klinischen Einsatz zu geben.

\section{Schlüsselwörter}

Episodisches Gedächtnis · Sprachfunktion · Reorganisation · Anteriore Temporallappenresektion · Funktionelle Konnektivität 

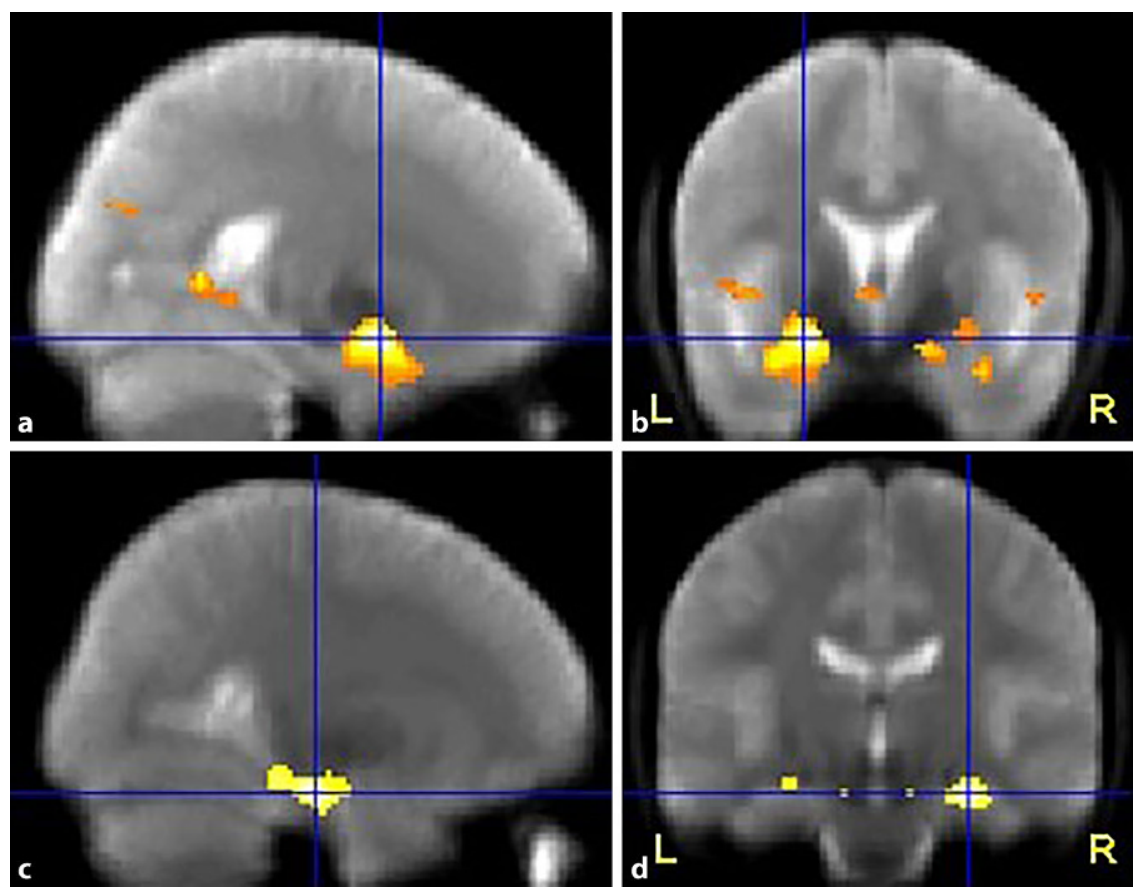

Fig. 1 Material specificity of verbal and visual memory encoding in healthy controls. a, b Word encoding, left hippocampal activation. c, d Face encoding, right hippocampal activation. Threshold $p<0.01$, uncorrected. Significant regions are superimposed onto an averaged normalized mean EP image from 30 healthy controls. (Adapted from Bonelli et al. 2010 [6])

advantage that they are generally most efficient in detecting differences between two conditions but interpretation of their contrasts does not allow one to account for subsequent memory effects. Eventrelated designs on the other hand are less powerful than block designs at detecting differences in two different brain stages and also more vulnerable to alterations in the hemodynamic response function; however, they have the main advantage that brain regions showing greater activation during encoding of different items that were subsequently remembered compared with items that were subsequently forgotten can be identified, representing the neural correlates of successful memory encoding [42].

\section{Prediction of memory decline}

Over the past years, many studies focused on the identification of prognostic indicators for risk of memory loss after ATLR. Besides the severity of hippocampal sclerosis (HS) on MRI, with less severe HS increasing the risk of memory decline [36], and preoperative performance on neuropsychological tests, with higher preoperative scores indicating a greater risk for postoperative decline [2, 15], language lateralization assessed by the IAP or language fMRI has been found helpful for predicting memory outcome [1]. These risk factors reflect the functional integrity of the resected temporal lobe and suggest that patients with residual memory function in the pathological hippocampus are at greater risk of memory impairment after ATLR. Other epilepsyrelated factors such as age of epilepsy onset and duration of epilepsy have also been identified as useful predictors of postoperative outcome [3].

Several studies have investigated the predictive value of fMRI for verbal memory decline $[24,27,29]$. Only a few fMRI studies have investigated visual memory after ATLR [24]. In patients with left HS, greater verbal memory encoding activity in the left hippocampus prior to surgery predicted the extent of verbal memory decline following left ATLR [24, 27-29]. These findings have since been replicated and extended to patients undergoing right ATLR [6, 24]. Using a material-specific memory-encoding paradigm in a large cohort of pa- tients with unilateral TLE, Bonelli et al. demonstrated that relatively greater ipsilateral anterior MTL activation was predictive of verbal and visual memory decline after left or right ATLR, while relatively greater posterior MTL activation was associated with better verbal and visual memory outcome [6]. In this study, memory asymmetry indices of anterior MTL activation had the strongest predictive value for verbal and visual memory decline compared with other epilepsyrelated variables. A prediction model comprising the aforementioned memory asymmetry index in combination with degree of language dominance and preoperative verbal memory performance correctly predicted verbal memory decline in all patients of the study. Prediction of visual memory decline was less accurate [6]. Taking these results further, Sidhu et al. demonstrated that predominantly left-sided activations within the frontal and medial temporal lobes correlated with significant verbal memory decline after left ATLR. They found a strong correlation between a memory laterality index (LI) using a mask in the left frontal and medial temporal lobes and regression-adjusted change in ability to learn a supra-span word list over serial presentations [33]. This demonstrated involvement of frontal networks in verbal learning processes that appear useful in predicting postsurgical memory change emphasizing the role of the networks involved in cognitive processes rather than of single structures.

To date, according to the practical guidelines provided by the American Academy of Neurology, the use of fMRI during presurgical evaluation may be considered for lateralizing memory functions in place of IAP in patients with MTLE (Level C); fMRI of verbal memory or language encoding should be considered for predicting verbal memory outcome (Level B), while fMRI using nonverbal memory encoding may be considered for predicting visuospatial memory outcomes (Level C; [34]). 

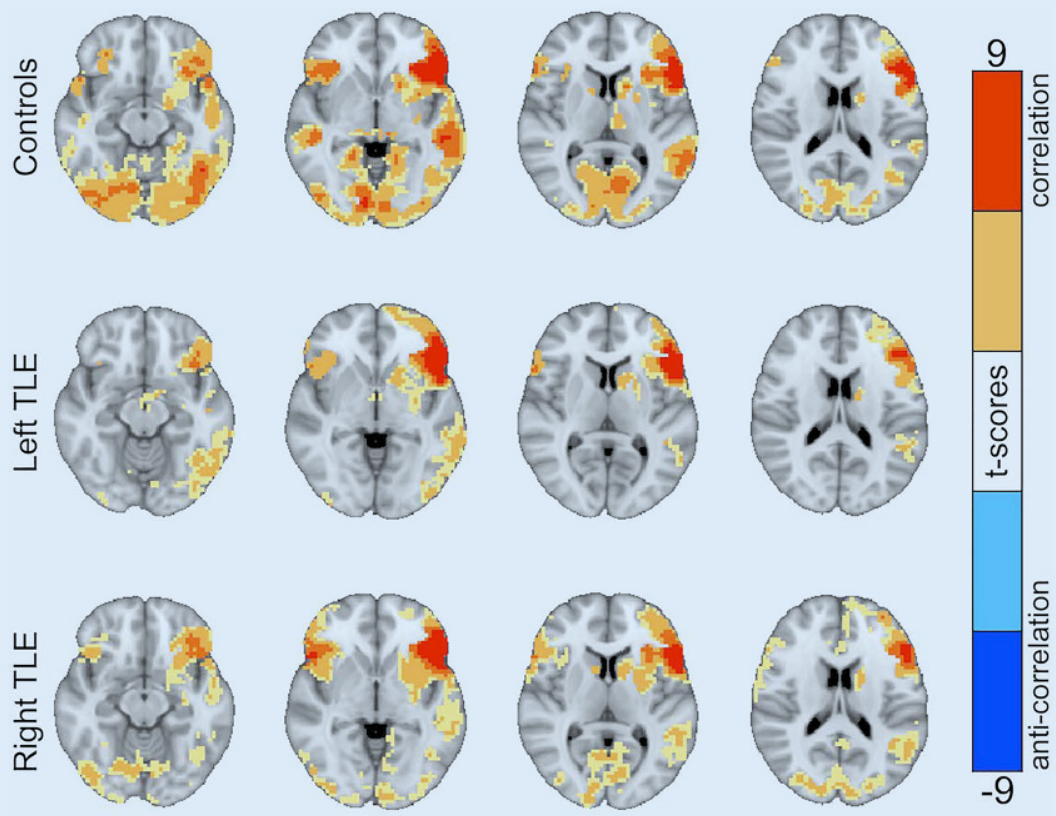

Fig. $2 \Delta$ Reduced functional connectivity of language-related areas is found in TLE patients compared with healthy controls. (Image courtesy of Karl-Heinz Nenning, Medical University of Vienna)

\section{Future perspectives: functional connectivity and connectome analyses}

The possibility to analyze functional connectivity (FC) in patients with epilepsy has significantly improved our understanding of seizure generation and propagation. The analysis of FC during cognitive tasks allows us to study the neuronal networks that sub-serve these tasks. In the past few years it has been demonstrated that FC alterations within the epileptic network but also in networks of cognitive functions such as language and memory are commonly observed (• Fig. 2). Left-sided TLE was associated with decreased FC within the expressive language network [43]. Specifically, FC was decreased in the left hemisphere irrespective of the epileptogenic focus [26] and within the prefrontal and frontotemporal networks [39], which was associated with impaired clinical language performance [39]. Results of a recent fMRI study using task-based (auditory and picture naming) FC analysis found that stronger FC of left posterior inferior temporal regions to bilateral anterior and posterior temporal lobe regions, as well as of bilateral inferior precentral gyrus and contralateral occipital cortex, was associated with better clinical naming performance in left and right TLE and in healthy controls. In left TLE, FC increased with later age of onset of epilepsy and shorter disease duration leading to a disrupted recruitment of temporal lobe networks ipsilateral to the seizure focus, which might account for naming deficits in TLE [37].

Within memory-specific networks, studies showed that in TLE, FC was reduced between the posterior cingulate and the epileptogenic hippocampus and increased between the posterior cingulate and the contralateral hippocampus $[19,22]$. In another study a visual scene-encoding task was used to evaluate memory function in healthy controls compared with patients with TLE. In this study, patients with left TLE demonstrated a significant decrease in FC to the inferior temporal, occipital, cingulate, and parietal cortices and the thalamus. In addition, the authors showed that orbital frontal activity correlated with structural measures of tract coherence in the fornix, which led to the suggestion that this might be the structural correlate of reduced FC [40]. Vollmar and colleagues reported similar observations of increased connectivity of motor and cognitive networks in patients with juvenile myoclonic epilepsy [41].

Connectome analysis is a relatively young and promising chapter in epilepsy imaging, which has just evolved over the past few years. In epilepsy patients, network analyses have the potential to explain cognitive deficits extending beyond seizure focus and to identify relevant reorganization patterns in individual patients. A key to the quantitative modeling of these relationships is capturing the connectome and its changes on the individual level. In TLE, studies investigating alterations of the structural [4] and functional [18] connectome are just beginning to emerge, suggesting that TLE has a widespread impact on structural and functional connectivity.

\section{Conclusion}

Functional MRI serves as a reliable method with which to visualize functional brain areas noninvasively, which allows us to study the effects of chronic epilepsy and epilepsy surgery on cognitive functions. Clinically, the method represents an important component in the presurgical work-up of TLE patients. Primarily, fMRI is applied to lateralize and localize language-related brain areas prior to surgery. Furthermore, the presurgical assessment of memory function by fMRI is recommended in patients suffering from a mesiotemporal seizure origin. For this purpose, general recommendations have been developed by the AAN and the ASFN.

The use of fMRI for FC analysis is another exciting area to study brain network dynamics. Functional connectivity can elicit neuronal networks that contribute to various cognitive tasks. It has been shown that cognitive impairment is often accompanied by reduced FC. Whether these methods may add to the prediction of postoperative cognitive outcome remains a topic of current research.

It is hoped that, in the near future, cognitive fMRI may be able to provide reliable results in individual patients, which when used in concert with other clinical, electrophysiological, and imaging results will not only predict postoperative 
cognitive decline in single patients but ultimately help prevent them.

\section{Practical conclusion}

- In order to assess language lateralization and localization by functional magnetic resonance imaging (fMRI), at least two tasks should be applied.

- Combining word generation and sentence completion tasks is most suitable for activating both expressive and receptive language areas.

- Object-naming and passive storylistening tasks represent alternative tasks used in order to assess language function in cognitively impaired or pediatric patients.

- During presurgical evaluation of patients with mesial temporal lobe epilepsy, fMRI may be considered to lateralize language and memory function and to predict postoperative language deficits; fMRI of verbal memory or language encoding should be applied to predict postsurgical verbal memory outcome.

- Functional connectivity and connectome analyses can elicit neuronal networks that contribute to various cognitive tasks. Whether these methods may add to the prediction of postoperative cognitive outcome remains a topic of current research.

\section{Corresponding address}

\section{Assoc.-Prof. PD Dr. Silvia Bonelli, PhD}

Department of Neurology, Medical University of Vienna

Währinger Guertel 18-20, 1090 Vienna, Austria silvia.bonelli@meduniwien.ac.at

Funding. Open access funding provided by Medical University of Vienna.

\section{Compliance with ethical guidelines}

Conflict of interest V.Schmidbauer and S. Bonelli declare that they have no competing interests.

For this article no studies with human participants or animals were performed by any of the authors. All studies performed were in accordance with the ethical standards indicated in each case.
Open Access This article is licensed under a Creative Commons Attribution 4.0 International License, which permits use, sharing, adaptation, distribution and reproduction in any medium or format, as long as you give appropriate credit to the original author(s) and the source, provide a link to the Creative Commons licence, and indicate if changes were made. The images or other third party material in this article are included in the article's Creative Commons licence, unless indicated otherwise in a credit line to the material. If material is not included in the article's Creative Commons licence and your intended use is not permitted by statutory regulation or exceeds the permitted use, you will need to obtain permission directly from the copyright holder. To view a copy of this licence, visit http://creativecommons.org/licenses/by/4.0/.

\section{References}

1. Baxendale S(2002) The role of functional MRI in the presurgical investigation of temporal lobeepilepsy patients: a clinical perspective and review. J Clin Exp Neuropsychol 24:664-676

2. Baxendale S, Thompson P, Harkness W et al (2006) Predicting memory decline following epilepsy surgery: a multivariate approach. Epilepsia 47:1887-1894

3. Baxendale S, Thompson PJ, Duncan JS (2008) Improvements in memory function following anterior temporal lobe resection for epilepsy. Neurology 71:1319-1325

4. Besson P, Dinkelacker V, Valabregue R et al (2014) Structural connectivity differences in left and right temporal lobe epilepsy. Neuroimage 100:135-144

5. Black DF, Vachha B, Mian A et al (2017) American Society of Functional Neuroradiology-Recommended fMRI Paradigm Algorithms for Presurgical Language Assessment. AJNR Am J Neuroradiol 38:E65-E73

6. Bonelli SB, Powell RH, Yogarajah M et al (2010) Imaging memory in temporal lobe epilepsy: predicting the effects of temporal lobe resection. Brain 133:1186-1199

7. Bonelli SB, Thompson PJ, Yogarajah $\mathrm{M}$ et al (2012) Imaging language networks before and after anterior temporal lobe resection-results of a longitudinal fMRI study. Epilepsia 53(4):639-650. https://doi.org/10.1111/j.1528-1167.2012.03433.

8. Davies KG, Bell BD, Bush AJ et al (1998) Naming decline after left anterior temporal lobectomy correlates with pathological status of resected hippocampus. Epilepsia 39:407-419

9. De Tisi J, Bell GS, Peacock JL et al (2011) The longterm outcome of adult epilepsy surgery, patterns of seizure remission, and relapse: a cohort study. Lancet 378:1388-1395

10. Duncan J (2009) The current status of neuroimaging for epilepsy. Curr Opin Neurol 22:179-184

11. Fernandez G, Specht K, Weis S et al (2003) Intrasubject reproducibility of presurgical language lateralization and mapping using fMRI. Neurology 60:969-975

12. Giussani C, Roux FE, Ojemann J et al (2010) Is preoperative functional magnetic resonance imaging reliable for language areas mapping in brain tumor surgery? Review of language functional magnetic resonance imaging and direct cortical stimulation correlation studies. Neurosurgery 66:113-120

13. Haag A, Knake S, Hamer HM et al (2008) The Wada test in Austrian, Dutch, German, and Swiss epilepsy centers from 2000 to 2005: a review of 1421 procedures. Epilepsy Behav 13:83-89

14. Helmstaedter C (2004) Neuropsychological aspects of epilepsy surgery. Epilepsy Behav 5(Suppl 1):S45-S55

15. Helmstaedter C, Elger CE (1996) Cognitive consequences of two-thirds anterior temporal lobectomy on verbal memory in 144 patients: a three-month follow-up study. Epilepsia 37:171-180

16. Janecek JK, Swanson SJ, Sabsevitz DS et al (2013) Language lateralization by $\mathrm{fMRI}$ and Wada testing in 229 patients with epilepsy: Rates and predictors of discordance. Epilepsia 54:314-322

17. Kunii N, Kamada K, Ota T et al (2011) A detailed analysis offunctionalmagneticresonanceimaging in the frontal language area: a comparative study with extraoperative electrocortical stimulation. Neurosurgery 69:590-596 (discussion 596-597)

18. Liao W, Ji GJ, Xu Q et al (2016) Functional connectome before and following temporal lobectomy in mesial temporal lobe epilepsy. Sci Rep 6:23153

19. Mccormick C, Quraan M, Cohn M et al (2013) Default mode network connectivity indicates episodic memory capacity in mesial temporal lobe epilepsy. Epilepsia 54:809-818

20. Middlebrooks EH, Ver Hoef L, Szaflarski JP (2017) Neuroimaging in epilepsy. Curr Neurol Neurosci Rep 17:32

21. Moosa ANV, Wyllie E (2017) Cognitive outcome after epilepsy surgery in children. Semin Pediatr Neurol 24:331-339

22. Pereira FR, Alessio A, Sercheli MS et al (2010) Asymmetrical hippocampal connectivity in mesial temporal lobe epilepsy: evidence from resting state fMRI. BMC Neurosci 11:66

23. Powell HW, Koepp MJ, Symms MR et al (2005) Material-specific lateralization of memory encoding in the medial temporal lobe: blocked versus eventrelated design. Neuroimage 27:231-239

24. Powell HW, Richardson MP, Symms MR et al (2008) Preoperative fMRI predicts memory decline following anterior temporal lobe resection. JNeurol Neurosurg Psychiatry 79:686-693

25. Powell HW, Richardson MP, Symms MR et al (2007) Reorganization of verbal and nonverbal memory in temporal lobe epilepsy due to unilateral hippocampal sclerosis. Epilepsia 48:1512-1525

26. Pravata E, Sestieri C, Mantini D et al (2011) Functional connectivity MR imaging of the language network in patients with drug-resistant epilepsy. AJNR Am J Neuroradiol 32:532-540

27. Richardson MP, Strange BA, Duncan JS et al (2006) Memory fMRI in left hippocampal sclerosis: optimizing the approach to predicting postsurgical memory. Neurology 66:699-705

28. Richardson MP, Strange BA, Duncan JS et al (2003) Preserved verbal memory function in left medial temporal pathology involves reorganisation of function to right medial temporal lobe. Neuroimage 20(Suppl 1):S112-S119

29. Richardson MP, Strange BA, Thompson PJ et al (2004) Pre-operative verbal memory fMRI predicts post-operative memory decline after left temporal lobe resection. Brain 127:2419-2426

30. Rosenow F, Luders H (2001) Presurgical evaluation of epilepsy. Brain 124:1683-1700

31. Sabsevitz DS, Swanson SJ, Hammeke TA et al (2003) Use of preoperative functional neuroimaging to predict language deficits from epilepsy surgery. Neurology 60:1788-1792

32. Sidhu MK, Stretton J, Winston GP et al (2013) A functional magnetic resonance imaging study 


\section{Leitthema}

mapping the episodic memory encoding network in temporal lobe epilepsy. Brain 136:1868-1888

33. Sidhu MK, Stretton J, Winston GP et al (2015) Memory fMRI predicts verbal memory decline after anterior temporal lobe resection. Neurology 84:1512-1519

34. Szaflarski JP, Gloss D, Binder JR et al (2017) Practice guideline summary: Use of $\mathrm{fMRI}$ in the presurgical evaluation of patients with epilepsy: Report of the Guideline Development, Dissemination, and Implementation Subcommittee of the American Academy of Neurology. Neurology 88:395-402

35. Towgood K, Barker GJ, Caceres A et al (2015) Bringing memory $\mathrm{fMRI}$ to the clinic: comparison of seven memory fMRI protocols in temporal lobe epilepsy. Hum Brain Mapp 36:1595-1608

36. Trenerry MR, Jack CR Jr., Ivnik RJ et al (1993) MRI hippocampal volumes and memory function before and after temporal lobectomy. Neurology 43:1800-1805

37. Trimmel K, Van Graan AL, Caciagli L et al (2018) Left temporal lobe language network connectivity in temporal lobe epilepsy. Brain 141:2406-2418

38. Trimmel K, Van Graan LA, Gonzalvez GG et al (2019) Naming fMRI predicts the effect of temporal lobe resection on language decline. Ann Clin Transl Neurol6(11):2186-2196. https://doi.org/10.1002/ acn3.50911

39. Vlooswijk MC, Jansen JF, Majoie HJ et al (2010) Functional connectivity and language impairment in cryptogenic localization-related epilepsy. Neurology 75:395-402

40. Voets NL, AdcockJE, Stacey Ret al (2009) Functional and structural changes in the memory network associated with left temporal lobe epilepsy. Hum Brain Mapp 30:4070-4081

41. Vollmar C, O'muircheartaigh J, Barker GJ et al (2011) Motor system hyperconnectivity in juvenile myoclonic epilepsy: a cognitive functional magnetic resonance imaging study. Brain 134:1710-1719

42. Wagner AD, Koutstaal W, Schacter DL (1999) When encoding yields remembering: insights from event-related neuroimaging. Philos Trans R Soc Lond B Biol Sci 354:1307-1324

43. Waites AB, Briellmann RS, Saling MM et al (2006) Functional connectivity networks are disrupted in left temporal lobe epilepsy. Ann Neurol 59:335-343

44. Woermann FG, Labudda K (2010) Clinical application of functional MRI for chronic epilepsy. Radiologe 50:123-130

\section{Hier steht eine Anzeige.}

\section{Springer}

